\title{
Design and Calibration of a High Volume Fogwater Collector
}

\author{
Gerald Pade, David Covert and Timothy LaRson \\ University of Washington, Department of Civil Engineering, Seattle, WA 98195 \\ 9 April 1986 and 15 October 1986
}

\begin{abstract}
An inertial impactor is described that is designed to actively collect fog droplets from a flowing airstream. This collector samples 60 liters of air per second with a theoretical $d_{50}$ currently set at $8 \mu \mathrm{m}$. Collector performance was assessed by generating a known number of 22 and $44 \mu \mathrm{m}$ water droplets tagged with $1 \mathrm{ppm}$ sodium fluorescein and injecting these droplets into a larger flow of polydisperse, untagged droplets immediately upstream of the collector. These large, chemically tagged droplets were found to be collected with $90 \%$ efficiency, in good agreement with theoretical estimates of collector performance. Between 3 and $8 \%$ water loss via evaporation was observed for relative humidities between $93 \%$ and $88 \%$, respectively.
\end{abstract}

\section{Introduction}

Careful measurement of fogwater chemistry is an important part of the ongoing research into the environmental consequences of "acid fog" (Hoffman, 1984; Hileman, 1983; Waldman et al., 1985). Numerous collector designs have been developed for sampling fog based upon the principle of inertial impaction (Castillo et al., 1983; Houghton, 1955; Jacob et al., 1985; Fuzzi et al., 1984; May 1945; Katz, 1980; Jacob et al., 1982; Jacob et al., 1984) yet few experimental calibrations have been performed (Katz, 1980; Jacob et al., 1982; Jacob et al., 1984). In this paper we discuss a new droplet impactor/collector that is safe, inexpensive and easy to operate, and capable of collecting up to $1 \mathrm{ml}$ of fogwater per minute in field tests. Droplets that impact upon the collection surfaces are subsequently collected in the sample vial with minimal evaporative losses.

\section{Design}

Cross-sectional schematics of the collector are shown in Figs. 1 and 2. The collector can be thought of as two separate assemblies: a removable collector assembly (impaction rods, hub, collector vial), which rests inside of a blower assembly (blower, inlet, stand). The collector assembly consists of a central $1.6 \mathrm{~cm}$ diameter teflon hub approximately $6 \mathrm{~cm}$ in length, in which two rows of twenty $0.17 \mathrm{~cm}$ holes have been drilled at a $45^{\circ}$ angle for the insertion of $9.5 \mathrm{~cm}$ long acrylic impaction rods. The lower $1.5 \mathrm{~cm}$ of the hub is cut into a 30 degree angle cone. Just below the top of this cut, three $0.32 \mathrm{~cm}$ holes are drilled out on a slight downward angle for the tripod which supports the collector assembly inside the blower inlet. The three acrylic support rods also hold a $3.5 \mathrm{~cm}$ plastic funnel attached to a plastic collection vial. A $1 \mathrm{~cm}$ diameter hole drilled into the side of the vial completes the airflow circuit through the second impaction stage. (If the hole is placed at or below the tip of the funnel, considerable water loss will occur.) A removable handle was made from $1 \mathrm{~cm}$ teflon stock and threaded for insertion into the top of the hub.

The blower assembly consists of a Rotron Centrimax blower (EG \& G model CX33A2G) suitably affixed to a camera tripod via a mounting plate. The inlet is constructed out of two automotive 4 bbl carburetor "velocity stacks" (Mr. Gasket Co., model 46). One of these "stacks" is inverted and cut to fit onto the entrance of the blower, while the other sits on top of it, forming a venturi shape.

Seats for the collector assembly tripod were constructed out of $1 \mathrm{~cm}$ teflon stock cut to $1 \mathrm{~cm}$ lengths and slotted for the support rods to sit in. The exact details of the supports for the collector assembly and the blower are not critical to the design of the collector. In addition, a small sheet metal deflector was attached to the outlet of the blower to prevent resampling of the exhaust.

Measurements of the velocity profiles in the impaction area were performed using a small pitot tube and micromanometer. An extra "velocity stack" was purchased, affixed, and appropriately tapped for making static pressure measurements and traverses with the pitot tube. The blower used operates on $110 \mathrm{ac}$ and is capable of pulling $259 \mathrm{~L} \mathrm{~s}^{-1}$ through the inlet (at 3.2 amp). However, with the collector assembly inserted the flow rate is reduced to $254 \mathrm{~L} \mathrm{~s}^{-1}$. This creates air velocities in the impaction area of from 14 to $18 \mathrm{~m}$ $\mathrm{s}^{-1}$, depending upon location (see Fig. 1).

As fog droplets enter the device, they are impacted upon the radial array of rods. Over the range of Weber numbers present in the impaction area $(\leqslant 150)$, droplet

(C) 1987 American Meteorological Society 


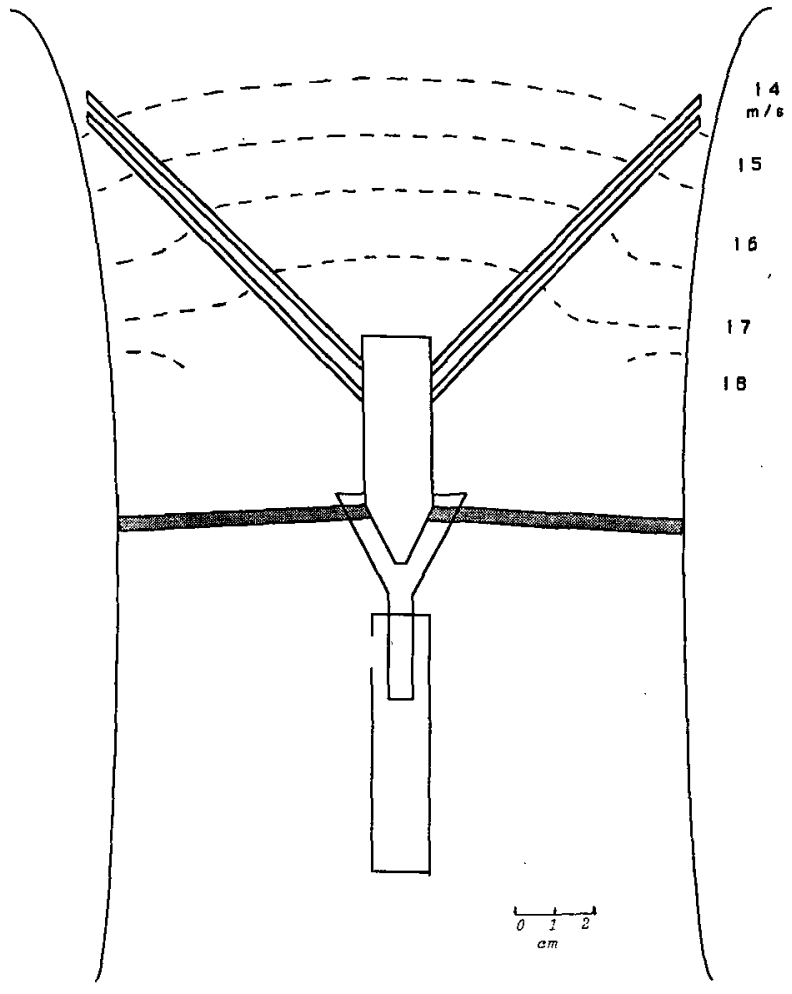

FIG. 1. Vertical cross section of collector. Dashed lines indicate lines of constant measured air velocity in $\mathrm{m} \mathrm{s}^{-1}$.

shattering is not expected. These droplets are then forced to the back side of the rods, where they rapidly coalesce. Once they reach a critical size $(\sim 1 \mathrm{~mm})$, aerodynamic drag causes the droplets to proceed rapidly toward the hub. Airflow between the hub and funnel accelerates these now much larger droplets through the funnel and into the collection vial. The inertia of these large drops completely prevents them from escaping with the airflow out the hole in the side of the collection vial. When the collection vial contains a sufficient sample volume $(2-10 \mathrm{ml})$, the vial is changed by attaching the handle and removing the collector assembly from the inlet. A clean collector vial is then attached to the collection assembly and the complete assembly reinserted into its seat.

\section{Calibration}

Calibration was performed by generating a precisely known number of monodisperse droplets chemically tagged with $1 \mathrm{ppm}$ sodium fluorescein and injecting these droplets into an artificial, polydisperse water fog immediately upstream of the impaction rods. A mass balance was then performed on the fluorescein. Aerosol generation was accomplished using a Berglund-Liu monodisperse aerosol generator (Thermo-Systems, Inc. model 3050). The vibrating orifice cover was modified to prevent aerodynamic turbulence above the impac- tion rods by machining a piece of plastic into a conical shape with provisions for a $1 \mathrm{~cm}$ I.D. thin wall stainless 'steel exit tube and a space charge neutralization section located next to the aerosol generator head. Aerosol charge neutralization was accomplished using $1.2 \mathrm{mil}-$ licurie strength alpha emission from the Polonium 210 elements of Staticmaster brushes (Nuclear Products Co. model 2U500). The Polonium-containing strips were arranged into a circle ( $4 \mathrm{~cm}$ diameter) about the jet in order to neutralize the negative charge acquired by the droplets during atomization, and to prevent excessive loss of droplets onto the exit tube.

The amount of fluorescein generated was verified by measuring the volumetric flow rate of the syringe pump, found to be in agreement within $2 \%$ of the stated nominal values. The loss of these monodisperse droplets to the wall of the exit tube was minimized by neutralization of the charge acquired during atomization. Additionally, the dispersion air flow was maintained at $25 \mathrm{~L} \mathrm{~min}^{-1}$ in order to keep the jet of droplets aimed straight down the vertically oriented exit tube. The wall loss was quantified by carefully rinsing the exit tube after each measurement, and was included in all efficiency calculations. At the conclusion of the experiment, the charge-neutralization section was rinsed and the fluorescein remaining found to be equivalent to less than $2 \%$ of the total amount generated.

Two transverses were made across the inlet at $1 \mathrm{~cm}$ intervals while maintaining a distance of approximately $6 \mathrm{~cm}$ above the impaction rods. Meanwhile, an ultrasonic nebulizer was producing a polydisperse, $\mathrm{pH}$ buffered water fog around the monodisperse droplets

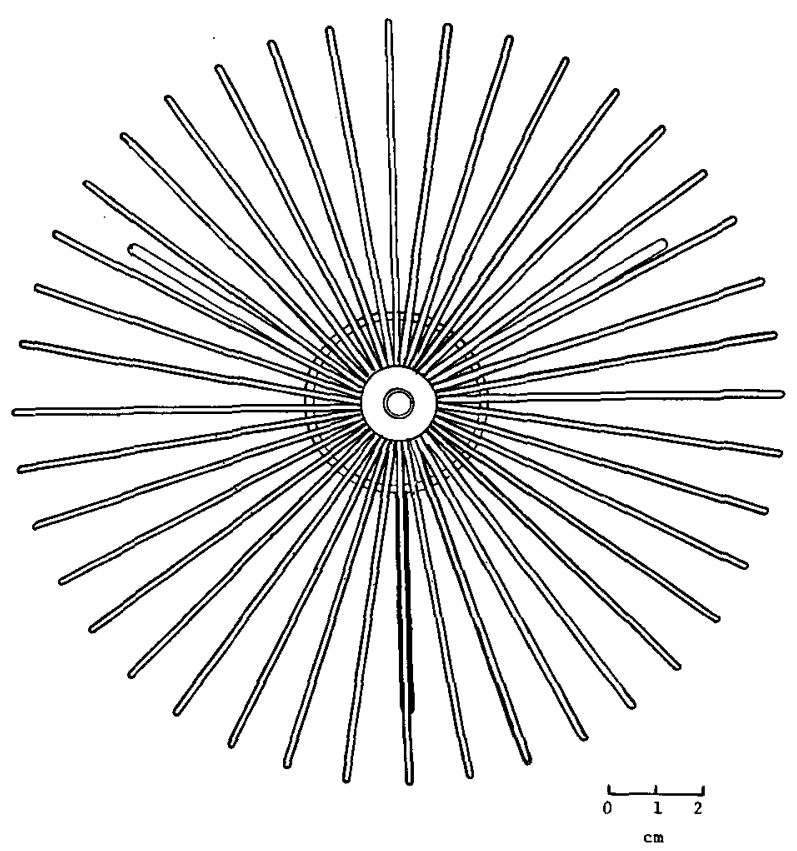

FIG. 2. Top view of collector. 
to prevent them from evaporating and to maintain realistic operating conditions. A Spectra Physics ruby red laser was used to illuminate the monodisperse aerosol and to warn the operator of possible jet failures. Each sample (12 total) took approximately 30 minutes. A Turner filter fluorometer (model 110) was used to determine the fluorescein concentrations.

Uncertainty in the mass of fluorescein collected in the sample vial and exit tube rinse could be attributed to one of two causes: the measurement of sample volume, and fluorescence. Sample volume could be measured to within $0.05 \mathrm{ml}$ and fluorescence to within 1 unit $\left(0.002 \mu \mathrm{g} \mathrm{ml}^{-1}\right)$. This uncertainty could account for less than a $2 \%$ error in collection efficiency.

As a check on the monodispersity of the droplets, a small air jet (provided with the aerosol generator) was used to deflect the stream of droplets exiting the orifice. The presence of the "satellite" droplet streams, indicative of doublets and triplets, were eliminated by tuning the sine-wave generator to a suitable frequency. Droplet diameters were both calculated and experimentally verified using a magnesium oxide-coated slide technique. The diameters of two different size droplets were calculated to be 22 and $44 \mu \mathrm{m}$ from measurements of syringe pump flow rate and vibration frequency. Microscopic analysis found them to be roughly 28 and $49 \mu \mathrm{m}$ respectively, although this latter method is somewhat uncertain as it involves indirect measurement of the splash pattern on powdered slides.

In addition to the above measurements, the theoretical collection efficiency of this device was predicted as a function of droplet diameter. As a basis for the

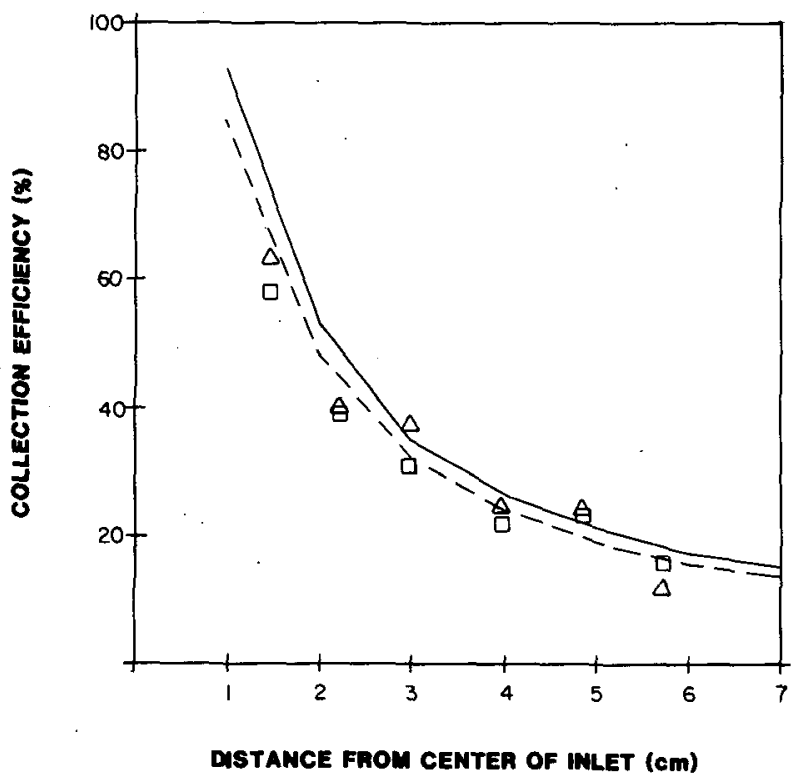

FIG. 3. Theoretical versus measured single rod collection efficiency as a function of radial distance from the center of the inlet: (dashed) potential flow theory; (solid) Wong and Johnstone; $\Delta 22 \mu \mathrm{m}$ droplets; $\square 44 \mu \mathrm{m}$ droplets).

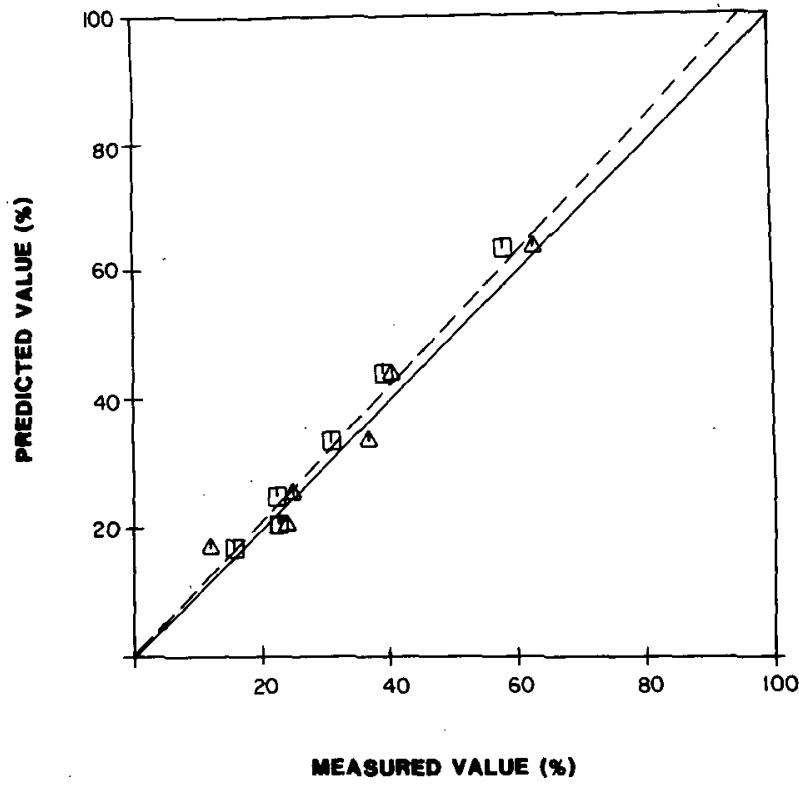

FIG. 4. Theoretical versus measured single rod collection efficiency. Solid line indicates perfect agreement, dashed line indicates best fit to data ( $R=0.98 ; \Delta 22 \mu \mathrm{m}$ droplets; $\square 44 \mu \mathrm{m}$ droplets).

predictions, the potential flow equations used by Langmuir and Blodgett (1945) for flow around cylinders were used. In addition, the measurements by Wong and Johnstone (1953) of the impaction of droplets onto cylinders were compared over a similar range of Stokes numbers (i.e., 1-20). (Stokes numbers for the droplets used in the calibration ranged from 10-100.) The problem of characterizing this device was greatly simplified by its radial symmetry. Seven equally spaced annular sections extending radially from the hub were defined, and the average impaction parameters in each section were determined. One of the most important parameters governing the overall collection efficiency of each section (and the device as a whole) is the fraction of air that is swept by the impaction rods. This is defined as the cross sectional area of the impaction rods normal to the airstream, relative to the total area of that section. These factors were combined with the individual rod collection efficiencies to obtain the collection efficiency of each section. Because the individual rod collection efficiency is droplet size-dependent, a total of 28 calculations were performed (four particle sizes for each section). These results are compared with the experimental data in Figs. 3 and 4. Both curves in Fig. 3 are based upon Stokes's numbers calculated for $20 \mu \mathrm{m}$ droplets moving at the respective velocities found in each section. In Fig. 4, the mean value of the two curves in Fig. 3 is compared with the mean of the experimentally obtained values using 22 and $44 \mu \mathrm{m}$ droplets.

The final step in the calculations involved a volumeweighted integration of the sectional collection effi- 
TABLE 1. Sampling results $(\mu \mathrm{M})$.

\begin{tabular}{cccccccc}
\hline \hline $\begin{array}{c}\text { Date } \\
\text { (mo/day/yr) }\end{array}$ & $\begin{array}{c}\text { Time } \\
\text { (LST) }\end{array}$ & $\mathrm{pH}$ & $\mathrm{SO}_{4}^{-}$ & $\mathrm{NO}^{-}$ & $\mathrm{Cl}^{-}$ & $\mathrm{Br}^{-}$ & $\mathrm{H}^{+}$ \\
\hline $1 / 2 / 85$ & $0955-1028$ & 3.93 & 174 & 154 & 317 & - & 117 \\
$1 / 3 / 85$ & $1031-1110$ & 3.73 & 179 & 172 & 297 & - & 186 \\
$1 / 3 / 85$ & $1120-1140$ & 3.32 & 474 & 593 & 298 & 4.3 & 479 \\
$1 / 3 / 85$ & $1140-1255$ & 3.17 & $\mathrm{NA}$ & $\mathrm{NA}$ & $\mathrm{NA}$ & $\mathrm{NA}$ & 676 \\
$1 / 3 / 85$ & $1505-1545$ & 2.90 & 951 & 1608 & 632 & 9 & 1259 \\
$1 / 3 / 85$ & $1546-1720$ & 3.03 & 513 & 910 & 279 & 5.4 & 933 \\
$1 / 3 / 85$ & $1721-1755$ & 3.50 & 271 & 471 & 245 & - & 316 \\
$1 / 3 / 85$ & $1800-1815$ & 3.71 & 245 & 366 & 264 & 3.9 & 195 \\
$1 / 3 / 85$ & $1820-1845$ & 3.75 & 237 & 308 & 252 & - & 178 \\
$1 / 4 / 85$ & $1024-1103$ & 3.69 & 123 & 128 & 97 & - & 204 \\
$1 / 8 / 85$ & $0807-0851$ & 4.28 & 111 & 186 & 380 & - & 52 \\
$1 / 8 / 85$ & $0853-0938$ & 4.12 & 227 & 297 & 266 & - & 76 \\
\hline
\end{tabular}

ciencies to obtain the overall collection efficiency of the sampler as a function of droplet size. The overall collection efficiency of the device is the summation of the products of sectional collection efficiencies and their corresponding volume weighting factors (airflow through section relative to total air flow). Results of these calculations are plotted in Fig. 5. Based on this calculation, we estimate that 60 liters of air per second are actively sampled by the rods.

Evaporative losses were estimated by raising the relative humidity of a small room to around $90 \%$ and then sampling a polydisperse fog generated by an ultrasonic nebulizer and chemically tagged with a known concentration of fluorescein. The volumetric flow rate of the fog exiting the nebulizer was only a small fraction of the total flow through the collector. Therefore, in this experiment, the collector was sampling some fog and mostly high relative-humidity room air. Increases in the fluorescence of the water sample relative to that which was nebulized would quantitatively indicate the amount of evaporative loss. (Any concentration occurring in the nebulization process would have appeared to be from evaporation, thus making our estimates a "worst case" scenario.) Evaporative losses of $8 \%, 6 \%, 4 \%$ and $3 \%$ were measured at relative humidities of $87 \%, 90 \%, 92 \%$ and $93 \%$, respectively. (Relative humidities were determined with a sling psychrometer.) At the minimum humidities encountered in "patchy" fogs $(>96 \%)$, the evaporative loss should be negligible.

The collector was field tested at a site located on the University of Washington campus in Seattle during the first week of January, 1985. Calculated liquid $\mathrm{H}_{2} \mathrm{O}$ content was within the range of $0.01-0.1 \mathrm{~g} \mathrm{~m}^{-3}$. Collection rates were in the range $0.1-1 \mathrm{ml} \mathrm{min}^{-1}$ depending upon the density of the fog. The twelve samples that were collected were marked by a decidedly greyish coloration. Chemical analysis of the major anions (Table 1) gave aqueous concentrations (e.g., $2.90 \leqslant \mathrm{pH} \leqslant$ 4.3; $\left.110 \mu \mathrm{M} \leqslant \mathrm{SO}_{4}{ }^{=} \leqslant 950 \mu \mathrm{M}\right)$ within the expected range reported elsewhere (Waldman, 1985; Fuzzi, 1984).

\section{Discussion and conclusions}

This design has several advantages. The radially symmetric inlet collects droplets with an efficiency independent of wind direction. The volumetric sampling rate of the air is well characterized, and the single droplet collection efficiency is relatively uniform as a function of location within the collector. The geometry is intended to actively collect the larger, coalesced droplets in the collection vial. Finally, power consumption is minimal and the collector surfaces can be easily cleaned and/or modified.

The strength of our collector assessment technique is in the use of aqueous droplets rather than solid par-

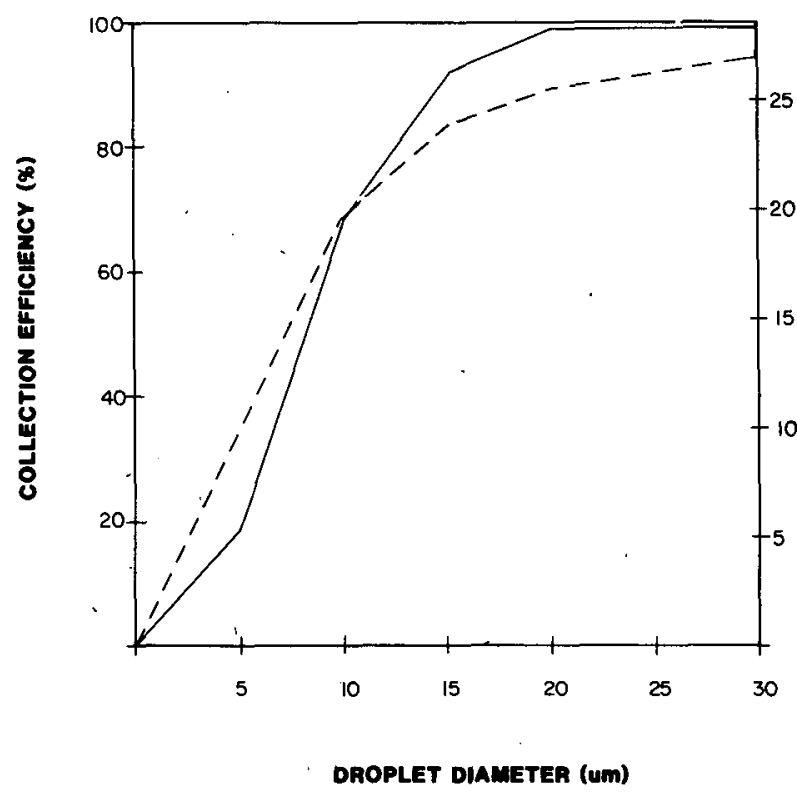

FIG. 5. Overall collection efficiency as a function of droplet size (right scale). [--- -.- --- potential flow theory as modified Langmuir and Blodgett (1945); - - Wong and Johnstone]. Scale on left considers only the air being swept by impactions rods. 
ticles (and vaseline-coated impaction surfaces) used by previous investigators (Jacob et al., 1984; Katz, 1980). Using solution droplets eliminates the uncertainties associated with particle "bounce" that may differ from solid to liquid particles. Our calibration results were found to be in excellent agreement with both theoretical and semi-empirical predictions $(R=0.98)$. However, the results are not sufficiently precise to discern whether the device's impaction efficiency is better described by potential flow theory or by analogy to the measurements of Wong and Johnstone. Lack of precision can be attributed to uncertainty in the exact radial location of impaction of the fluorescein-containing droplets. Although the aerosol release point was known, the exact point of impaction had to be estimated by analysis of the streamlines defined by the droplet stream from the nebulizer.

Evaporation of impacted droplets was shown to be negligible, being only $3 \%$ at $93 \% \mathrm{RH}$. This was to be expected based upon the design of the collector. Because the impacted droplets rapidly coalesce into progressively larger drops and quickly reach the sample vial where only a small surface area is exposed, the opportunity for evaporation is minimized. In addition, there is not enough kinetic energy in the droplets prior to impaction to cause significant droplet temperature rise and subsequent evaporation immediately after impaction.

The design of this sampler can be modified to suit various applications. To automate the device, the removable collection vial can be replaced by a piece of tubing leading from the funnel through an appropriately located hole in the inlet to a small test tube carousel. The overall collection efficiency of the device (governed primarily by the fraction of air swept) can be increased by adding an extra one or two rows of impaction rods. If a lower size cut is desired, this can be achieved by decreasing the impaction rod diameter, or to a lesser degree, by simply shortening the length of the rods. This would allow one to study the droplet size dependent chemistry of fog.
Acknowledgments. We thank William Zoller for providing chemical analysis. This project was supported by the Washington State Department of Ecology under contract number C0085065 and the U.S. EPA under Grant B-810879.

\section{REFERENCES}

Castillo, R., J. Jiusto and E. McLaren, 1983: The pH and ionic composition of stratiform cloud water. Atmos. Environ., 17, 14971505.

Fuzzi, S., R. Castillo, J. Jiusto and G. Lala, 1984: Chemical composition of radiation fog water at Albany, New York, and its relationship to fog microphysics. J. Geophys. Res., 89, 71597164.

Hileman, B., 1983: Acid fog: new findings widen the range of acid deposition phenomena. Proc. of the Air Pollution Control Assoc. Specialty Conf. on Meteorology and Acid Deposition, Hartford, 46-56.

Hoffmann, M., 1984: Comment on acid fog. Environ. Sci. Technol., 18, 61-64.

Houghton, H., 1955: On the chemical composition of fog and cloud water. J. Meteor., 12, 355-357.

Jacob, D., R. Flagan, J. Waldman and M. Hoffmann, 1982: Design and calibration of rotating arm collectors for ambient fog sampling. Proc. IV Int. Conf. on Precipitation Scavenging, Dry De position, and Resuspension, Santa Monica,

- , R. Wang and R. Flagan, 1984: Fogwater collector design and characterization. Environ. Sci. Technol., 18, 827-833.

- - J. Waldman, M. Haghi, M. Hoffmann and R. Flagan, 1985: Instrument to collect fogwater for chemical analysis. Rev. Sci. Instrum., 56, 323-333.

Katz, U., 1980: A droplet impactor to collect liquid water from laboratory clouds for chemical analysis. Commun. VIII Conf. Int. sur la Physique des Nuages, Clermont-Ferrand, France, 697700 .

Langmuir, I., and K. Blodgett, 1945: A mathematical investigation of water droplet trajectories. Tech. Rep. 541B, U.S. Army Air Force, Washington, D.C.

May, K., 1945: The cascade impactor: an instrument for sampling coarse aerosols. J. Sci. Instrum., 22, 187-195.

Waldman, J., J. Munger, D. Jacob and M. Hoffman, 1985: Chemical characterization of stratus cloudwater and its role as a vector for pollutant deposition in a Los Angeles pine forest. Tellus, 37B, 91-108.

Wong, J., and H. Johnstone, 1953: Collection of aerosols by fiber mats, Rep. No. 11, University of Illinois Experimental Station. 